\title{
A prefilled injection device for outreach tetanus immunization by Bolivian traditional birth attendants ${ }^{1}$
}

\author{
Rosario Quiroga, ${ }^{2}$ Percy Halkyer, ${ }^{3}$ Fernando Gil, ${ }^{4}$ \\ Carib Nelson, ${ }^{5}$ and Debra Kristensen ${ }^{5}$
}
ABSTRACT This study evaluated the performance, acceptability, and appropriateness of a new, single-use, prefilled injection device called UniJect ${ }^{\mathrm{TM}}$ for an outreach immunization application. ${ }^{6}$ Between April and June 1995, UniJect devices were used by 36 traditional birth attendants to admin- ister tetanus toxoid injections to 2240 pregnant women during routine, antenatal home visits in the Northern, Ichilos, and Warnes Districts of Santa Cruz, Bolivia. Because tetanus toxoid is relatively heat stable, the traditional birth attendants were able to keep the tetanus toxoid- filled UniJect devices in their homes for up to one month without refrigeration. The devices were stored, transported, and disposed of in an outreach carrier designed to reduce the risks of improper handling and disposal.
Data were collected from injection recipients, traditional birth attendants, and supervisors via observation, questionnaires, and post-study interviews. The performance of the UniJect device and its acceptability among all groups was very high. The traditional birth attendants used UniJect properly and safely; there were no reports or observations of device misuse, reuse, or needle-stick. Advantages cited included the fact that the device required no assembly, offered assured sterility, and reduced vaccine wastage sometimes associated with multi-dose vials. The ability to store and transport the vaccine-filled devices without ice also greatly simplified logistics.

\footnotetext{
Reprint requests and other correspondence should be addressed to Debra Kristensen, Program for Appropriate Technology in Health, 4 Nickerson Street, Seattle, Washington 98109, U.S.A.

2 Ministerio de Desarrollo Humano, Secretaría Nacional de Salud, La Paz, Bolivia.

3 Consultant, Expanded Program on Immunization, Pan American Health Organization, La Paz, Bolivia.

4 Consultant, Expanded Program on Immunization, Pan American Health Organization, Santa Cruz, Bolivia.

5 Program for Appropriate Technology in Health, Seattle, Washington 98109, U.S.A.

6 UniJect is a trademark of Becton Dickinson and Company. The device was developed by the Program for Appropriate Technology in Health with support from the United States Agency for International Development Technologies for Health (HealthTech) project.
}

Many immunization programs today struggle to achieve two objectives: extending coverage to reach underserved populations and improving injection safety $(1,2)$. Innovative methods are being explored to increase immunization coverage, including use of home visits (3), enlistment of community-based health workers to deliver injections (4), and use of heat-stable vaccines in remote areas without the support of cold-chain equipment and supplies $(5,6)$. At the same time, the emergence of human immunodefi- ciency and hepatitis B viruses as major public health concerns has prompted health agencies to adopt more stringent policies designed to prevent the improper reuse of needles and syringes (1). The use of autodestruct syringes is one mechanism employed to minimize injection risks $(1,7)$. These devices virtually eliminate the possibility of patient-to-patient transmission of bloodborne infections through reuse. However, they do not address some of the important needs of immunization outreach programs: 
Ease-of-use. Delivery of vaccine with autodestruct syringes requires the same steps as delivery with reusable or disposable syringes. An easier-to-use device could allow a wider range of community-based health workers to deliver injections.

Assurance that a dose of vaccine and a sterile syringe and needle will be available for each patient. Supplies of vaccine must be coordinated with supplies of injection equipment for outreach. Diversion of needles and syringes to other uses, and unbalanced supplies are problems that can be exacerbated at the periphery of the distribution chain.

Reduction of vaccine wastage. Unused vaccine in multi-dose vials must often be discarded resulting in high wastage rates and reluctance on the part of vaccinators to open a new vial for only one or two patients, thereby missing immunization opportunities.

In order to address these concerns, a prefilled, single-use injection deviceUniJect-was developed by the Program for Appropriate Technology in Health (see note 6, p. 20).

Bolivia has made significant progress in increasing tetanus toxoid coverage in recent years, and traditional birth attendants (TBAs) began training to give tetanus toxoid immunizations in their communities in 1990. Pan American Health Organization (PAHO) and Bolivian Ministry of Health officials decided to test UniJect to determine if it could serve as an appropriate device to allow TBAs to safely immunize women in their homes.

\section{MATERIALS AND METHODS}

\section{Injection device}

UniJect consists of a plastic disposable syringe that is prefilled with a single dose of medicament (Figure 1). The medicament is enclosed in a sealed reservoir, and a permanent needle is
FIGURE 1. UniJect ${ }^{\mathrm{TM}}$ prefilled, single-use injection device

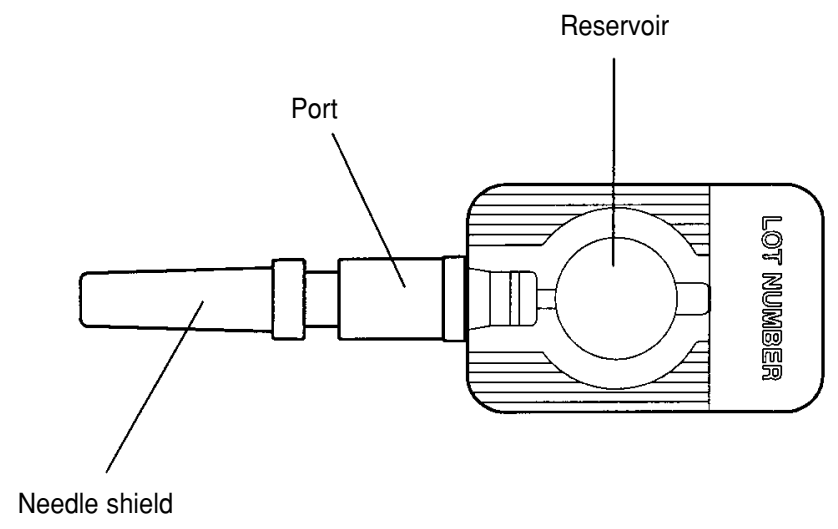

Actual size attached. To use UniJect, the device is removed from its foil pouch and activated by pushing the needle shield and port together. The needle shield is then removed, and the needle is inserted into the injection site. The dose is delivered by squeezing the reservoir until it collapses.

The device has several tamper-proof features: the reservoir cannot be resealed if opened or penetrated; a small, internal valve located between the reservoir and needle prevents refilling through the needle; and the needle cannot be removed from the device after use. This study used $0.5 \mathrm{~mL}$ UniJect devices with 23-gauge, $20 \mathrm{~mm}$ needles.

To date, the UniJect device has been successfully used in studies to deliver prostaglandin for prevention of postpartum hemorrhage in a hospital setting (8) and an injectable contraceptive in clinic settings (9).

\section{Vaccine}

The tetanus toxoid was produced and filled into the UniJect devices by Statens Seruminstitut, Copenhagen, Denmark, and subjected to standard release testing procedures. The expiration date for the tetanus toxoid-filled UniJect devices was January 1997. The filled devices were kept under normal cold-chain conditions during transport and storage at the national, district, and health center levels, but then were taken out of the cold chain for up to one month for use by the TBAs. The TBAs were asked to store the UniJects in their homes and carry them in an outreach carrier on antenatal visits. The average high temperature in Santa Cruz ranges from $24^{\circ}$ to $31^{\circ} \mathrm{C}$ (R. Victurine, personal correspondence, 1992). Because tetanus toxoid shows an insignificant decrease in potency when stored for 2 to 6 months at $37^{\circ} \mathrm{C}(10)$, it was considered safe to store the tetanus toxoid at ambient temperatures for one month. The TBAs were given special instructions about not exposing the vaccine to high temperatures such as fire or direct sunlight during storage or transport.

\section{Outreach carriers}

Special consideration was given to the safe disposal of used UniJect devices, since immunizations were administered outside the supervision of conventional health care facilities. A reusable outreach carrier with two internal compartments was developed and used in the study. One compartment stored tetanus toxoid-filled UniJect devices and the other held a removable cardboard disposal box into which used devices were inserted immediately after injection. On a 
monthly basis, TBAs returned to the health center to receive a new supply of UniJect devices and a new disposal box. Old disposal boxes were turned over to supervisors for incineration.

\section{Study participants}

TBAs from rural areas within the Northern, Ichilos, and Warnes districts in Bolivia's Santa Cruz region used UniJect to administer tetanus toxoid to pregnant women during routine, antenatal home visits. Two-thirds of the TBAs were female and one-third were male. Approximately half had no prior injection experience. Training activities were combined with a national tetanus awareness campaign to minimize disruption to ongoing immunization activities. Each TBA received a day of training focused specifically on UniJect, which included an opportunity to deliver several mock injections. After the initial training session, most TBAs delivered their first two or three UniJect injections while under direct supervision. A nurse supervisor was identified for each district and was responsible for study oversight and training of community nurse supervisors. One or two supervisors were identified for each community participating in the study. Their responsibilities included TBA training and supervision, distribution of supplies, disposal-box collection and incineration, and data collection.

\section{Data collection}

Data on the performance, acceptability, and appropriateness of the UniJect device were collected using three report forms, all completed by community supervisors. These comprised an observation checklist-completed while observing TBAs administering injections; a questionnaire to solicit TBA impressions of the device and outreach carrier; and a questionnaire to gather feedback from injection recipients. At the end of the study, an independent evaluator also visited participants in each study district and conducted in-depth interviews and group discussions with TBAs, and community and district supervisors. Efforts were made to obtain data from study participants in multiple communities within 2 of the 3 districts involved in the study.

\section{RESULTS}

Thirty-six TBAs administered 2240 UniJect injections between April and June 1995 (Table 1). In addition, supervisors administered questionnaires to 17 TBAs and 30 injection recipients, and observed 88 injections given by 14 TBAs. Further, 6 of 36 TBAs and 7 of 9 supervisors participated in in-depth interviews at the end of the study.

The ability of the UniJect device to function according to its design was evaluated. Indicators included an in- tact, vaccine-filled device, i.e., the presence of all parts; the ability of the device to inject the vaccine; and whether or not the device could be reused. Of the 2240 UniJects used in the study, only 5 (0.2 percent) did not function properly. Three were received empty and two would not expel the vaccine after being activated. There were no reports of attempted reuse.

Data gathered through TBA observation indicated that very few of the TBAs experienced difficulty in opening the package, activating the device, or delivering the injection (Table 2). Many of the TBAs initially had difficulty removing the needle shield; however, their difficulty diminished with experience. Most TBAs and their supervisors noted that delivering injections with UniJect was faster and easier than with standard syringes. The performance of the outreach carrier was satisfactory to almost all the TBAs who used it.

No needle-stick incidents were reported by the TBAs or recorded during the 88 injections observed by the supervisors, although some TBAs incorrectly recapped the devices before disposal. There were no reported problems with disposal of used injection devices, and outreach carrier users commented that they felt comfortable storing UniJect devices in their homes.

Supervisors administered questionnaires to 30 pregnant women who received tetanus toxoid injections with

TABLE 1. Participants, study on outreach tetanus immunization. Santa Cruz, Bolivia, April-June 1995

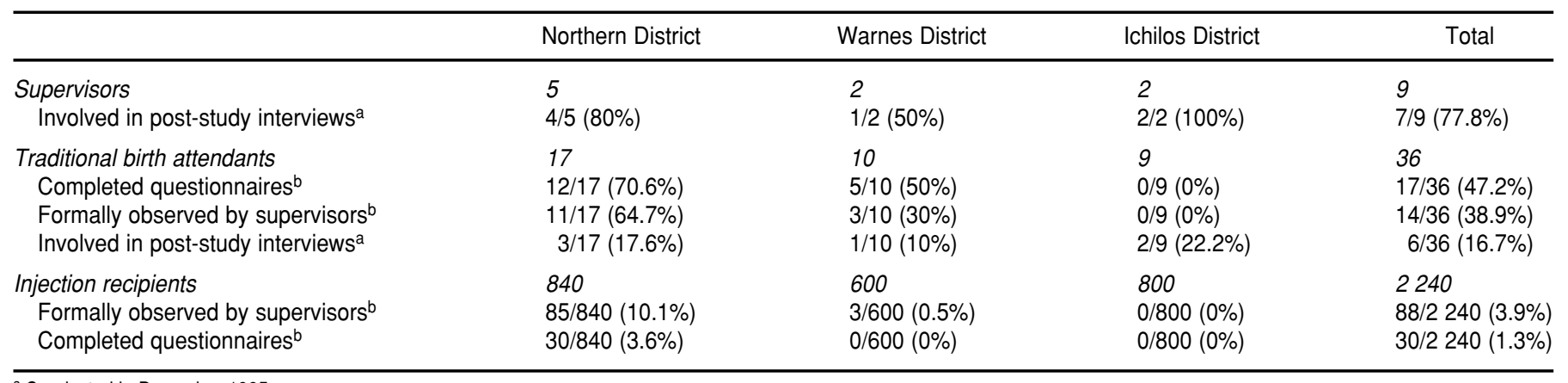

\footnotetext{
a Conducted in December 1995.

b Conducted between April and June 1995.
} 
TABLE 2. Results from checklists completed by supervisors observing traditional birth attendants (TBAs) giving injections with UniJect devices. Santa Cruz, Bolivia, April-June 1995

\begin{tabular}{lccc}
\hline \multicolumn{1}{c}{ Action evaluated-Did the TBA } & Always & Sometimes & Never \\
\hline Open the foil pouch easily? & $13 / 14$ & $1 / 14$ & $0 / 14$ \\
& $(93 \%)$ & $(7 \%)$ & $(0 \%)$ \\
Properly activate the UniJect device? & $14 / 14$ & $0 / 14$ & $0 / 14$ \\
& $(100 \%)$ & $(0 \%)$ & $(0 \%)$ \\
Verify that the port and needle shield were joined & $12 / 14$ & $1 / 14$ & $1 / 14$ \\
after activation? & $(86 \%)$ & $(7 \%)$ & $(7 \%)$ \\
Remove the needle shield without difficulty? & $5 / 14$ & $9 / 14$ & $0 / 14$ \\
& $(36 \%)$ & $(64 \%)$ & $(0 \%)$ \\
Remove the needle shield without expelling vaccine? & $13 / 14$ & $1 / 14$ & $0 / 14$ \\
& $(93 \%)$ & $(7 \%)$ & $(0 \%)$ \\
Clean the injection site? & $14 / 14$ & $0 / 14$ & $0 / 14$ \\
& $(100 \%)$ & $(0 \%)$ & $(0 \%)$ \\
Insert the device with a proper downward angle? & $12 / 13$ & $1 / 13$ & $0 / 13$ \\
& $(92 \%)$ & $(8 \%)$ & $(0 \%)$ \\
Squeeze the reservoir completely to deliver the entire & $14 / 14$ & $0 / 14$ & $0 / 14$ \\
dose of vaccine? & $(100 \%)$ & $(0 \%)$ & $(0 \%)$ \\
Dispose of the device without recapping?a & $8 / 13$ & $5 / 13$ & $0 / 13$ \\
Use the UniJect device without needle stick? & $(62 \%)$ & $(38 \%)$ & $(0 \%)$ \\
Dispose of the device properly? & $14 / 14$ & $0 / 14$ & $0 / 14$ \\
& $(100 \%)$ & $(0 \%)$ & $(0 \%)$ \\
\end{tabular}

${ }^{a}$ Information was not provided for one of the TBAs observed.

UniJect to gather feedback on their experience and impressions of the device. Although some initial hesitation was reported due to unfamiliarity with UniJect, over 90 percent said that they were not nervous or only slightly nervous about receiving a UniJect injection, and over 80 percent said that UniJect's appearance did not cause them any anxiety, in part because of its small size. The majority described the UniJect injection as "not painful" and less painful than previous injections that they had received (see Table 3). All stated that they would agree to a future UniJect injection; but 41 percent agreed with some reservations. Their reservations were not specific to UniJect, however, but reflected general concerns about the injections being performed properly and without pain or bleeding.

All TBAs who completed questionnaires $(n=17)$ commented on UniJect's simplicity, speed, and ease-of-use. The primary features that they said contributed to its simplicity and speed included having the vaccine prefilledwhich eliminated the need to load the syringe, and being able to store and transport the devices outside of the cold chain. Several TBAs with previous injection experience also commented that UniJect reduced vaccine wastage. TBAs who had not had previous injection experience said they felt increased respect and acceptance from the communities in which they worked during the UniJect trial. The TBAs also reported increased satisfaction and efficiency in their work due to their clients' favorable opinions about UniJect.

All supervisors who were interviewed $(n=7)$ stated that UniJect was used properly and easily by TBAs. They described UniJect as an appropriate tool for involving TBAs in immunization programs, resulting in in- creased community acceptance of local TBAs. Supervisors noted that UniJect use would simplify storage and distribution of vaccine supplies and injection equipment and reduce vaccine wastage. In addition, UniJect devices would be less likely to be stolen since they cannot be refilled or reused. Most supervisors stated that the outreach carrier was useful for UniJect storage, transport, and safe disposal. Some requested that the carrier be bigger to accommodate more UniJects and to reduce TBA travel time to resupply. All districts participating in the study issued unsolicited requests to central authorities for continued use of UniJect.

\section{DISCUSSION}

UniJect was used safely and effectively by TBAs, regardless of past injection experience, to administer tetanus toxoid to pregnant women in rural Bolivia. UniJect was shown to be reliable, easy to use, faster than a standard syringe, and well accepted by vaccinators, supervisors, and injection recipients.

Because a significant number of TBAs had difficulty removing the needle shield, design refinements were made to address this issue. Overall, the device functioned according to its design-efficiently delivering the dose of vaccine while preventing attempts at needle and syringe reuse.

The risk of contracting an infectious disease from unsafe injection practices is not limited to the person who receives the injection: bloodborne diseases can be transmitted from patient to patient from injections with contaminated equipment; patient to health care worker through accidental needle sticks; and patient to community through improper disposal of injection equipment $(2,11)$. During this study, no attempted reuse of UniJect devices was reported. While no needle-stick injuries were observed, a few TBAs were observed recapping used needles. Future training should emphasize the risk of needle-stick when recapping and should strongly discourage the practice. 
TABLE 3. Results of supervisor interviews with 30 injection recipients. Santa Cruz, Bolivia, April-June 1995

\begin{tabular}{ll}
\hline \multicolumn{1}{c}{ Interview question } & \multicolumn{1}{c}{ Responses } \\
\hline Was the UniJect injection painful? & Not painful: $16 / 30(53 \%)$ \\
& Slightly painful: $9 / 30(30 \%)$ \\
& Moderately painful: $5 / 30(17 \%)$ \\
& Very painful: $0 / 30(0 \%)$ \\
Was the UniJect injection more or less painful than & Less painful: $15 / 30(50 \%)$ \\
previous injections that you have received? & Similar: $3 / 30(10 \%)$ \\
& More painful: $2 / 30(7 \%)$ \\
& Could not compare: $10 / 30(33 \%)$ \\
Would you agree to receiving a UniJect injection in & Yes: $16 / 27(59 \%)$ \\
the future? & Yes, but with reservation: $11 / 27(41 \%)$ \\
\end{tabular}

a Information was not provided for 3 injection recipients.

The outreach carrier provided a safe method for syringe transport and disposal, and proper disposal procedures were followed during the study. The World Health Organization (WHO) recommends that used syringes be incinerated (2). UniJect contains only about 25 percent of the total amount of plastic used in standard disposable syringes. This plastic can be incinerated and does not emit toxic fumes such as those emitted by incineration of the rubber stopper commonly found in disposable syringes.

Finally, because UniJect is prefilled with a specific vaccine and is unusable with any other medicament, it is unlikely to be diverted to other uses. This improves the safety of the immunization program and decreases risks to the general community.

Acceptability and interest in continued UniJect use among injection recipients, vaccinators, and supervisors was very high, and program managers expressed interest in procuring UniJect for nationwide immunization programs. UniJect was shown to be highly appropriate for use by TBAs given the device's prefilled format and simplicity. Empowering TBAs to give injections was cited by Bolivian health officials as an effective method of increasing immunization coverage.

The UniJect study was unique because Bolivian TBAs were permitted to take vaccine out of the cold chain, store it in their homes, and travel di- rectly to immunization sessions within their community without having to travel to a health center first to pick up supplies. In addition, since insulated vaccine carriers and ice were not needed, the costs and complexity of conducting outreach were greatly reduced. According to the TBAs in the study, UniJect's single-dose format made it particularly appropriate for use outside the cold chain. Other advantages to removing certain heatstable vaccines (such as tetanus toxoid) from the cold chain include reducing the risk of vaccine damage due to freezing and increasing refrigerator space for heat-sensitive vaccines at the periphery of the distribution system.

As in many countries, current policy in Bolivia requires that open multidose vaccine vials be discarded at the end of the day, regardless of the number of doses remaining. Several supervisors confirmed that this policy results in high wastage rates with 20-dose vials of tetanus toxoid. While actual wastage figures were not available for Bolivia, vaccine wastage rates have been reported for other countries, e.g., 58 percent for vaccine supplied worldwide by the United Nations Childrens Fund (UNICEF) (6). UniJect could play an important role in reducing vaccine wastage in community outreach settings as it eliminates the need to discard unused vaccine.

Because tetanus toxoid is not yet commercially available in UniJect, it is difficult to assess product cost. However, since tetanus toxoid is an inexpensive vaccine, procurement in UniJect is likely to cost more per dose than procurement in multi-dose vials with separate syringes. On the other hand, use of UniJect may result in increased immunization coverage, reduced vaccine wastage, and simplified logistics. Long-term cost savings also might be achieved due to reduced transmission of bloodborne infections leading to reduced expenditures associated with disease treatment. A detailed cost/ benefit analysis is the logical next step before widespread introduction of tetanus toxoid in UniJect is considered. Costs of disposal systems such as the outreach carrier also need to be evaluated.

Several important operational issues were identified during the course of the trial, which need to be addressed before wide-scale introduction of vaccines in UniJect. First, although health workers expressed an interest in using UniJect for all immunizations, the device currently accommodates only 0.5 $\mathrm{mL}$ or $1.0 \mathrm{~mL}$ doses of non-lyophilized vaccines such as tetanus toxoid, tetanus-diphtheria, diphtheria-pertussis-tetanus, and hepatitis B. Second, cold-chain capacity should be carefully considered prior to UniJect introduction since its storage-volume per dose is higher than that of a multi-dose vial. Volume requirements and delivery schedules may need to be adjusted to ensure that sufficient cold-storage space is available at all levels of the distribution system. Third, establishing reliable supply lines for both UniJects and disposal boxes is critical to ensuring adequate vaccine supply and proper UniJect disposal. Finally, phased or pilot introduction of UniJect is recommended so that important operational issues can be identified and addressed. These issues likely will include training, cold chain impact, resupply, disposal, and cost.

Acknowledgments. We wish to thank the Pan American Health Organization, the Bolivian Ministry of Health, and the United States Agency 
for International Development for their support of this study; Statens Seruminstitut for provision of the vaccine, conducting a pilot fill of vaccine into UniJect devices, and laboratory analyses of the product; Horizon Medical, Inc., for assistance with the pilot filling operation; Dr. Amparo Pinzón for oversight of training and data collection; and Ms. Amie Bishop, Ms. Rebecca Fields, and Dr. Vivien Tsu for manuscript review.

\section{REFERENCES}

1. Henderson RH. Vaccination: successes and challenges. In: Cutts FT, Smith PG, eds. Vaccination and world health. England: John Wiley \& Sons Ltd; 1994:3-16.

2. Aylward B, Lloyd J, Zaffran M, McNair-Scott $R$, Evans P. Reducing the risk of unsafe injections in immunization programmes: financial and operational implications of various injection technologies. Bull World Health Organ 1995;73:531-540.

3. Brugha RF, Kevany JP. Maximizing immunization coverage through home visits: a controlled trial in an urban area of Ghana. Bull World Health Organ 1996;74:517-524.

4. Pan American Health Organization. PAHO close to eliminating neonatal tetanus from the Americas [press release]. March 20, 1997.
5. World Health Organization. Vaccine vial monitor and opened vial policy. Geneva: WHO; 1996.

6. World Health Organization, Expanded Programme on Immunization. Report of 1993 Technet Conference, 93.1.

7. Samuels M, Koop C, Hartsock P. Single-use syringes. N Eng J Med 1991;324:996-997.

8. Abdel-Aleem H, Abol-Oyoun EM, Moustafa SAM, Kamel HS, Abdel-Wahab HA. Carboprost trometamol in the management of the third stage of labor. Int $J$ Gynecol Obstet 1993;42:247-250.

9. Bahamondes L, Marchi NM, Cristofoletti MD, Nakagava HM, Pellini E, Araujo F, et al. UniJect as a delivery system for the once-a-month injectable contraceptive Cyclofem in Brazil. Contraception 1996;53:115-119.
10. Galazka A. Stability of vaccines. World Health Organization, Expanded Programme on Immunization; 1989.

11. Hu DJ, Kane MA, Heymann DL. Transmission of HIV, hepatitis B virus, and other bloodborne pathogens in health care settings: a review of risk factors and guidelines for prevention. Bull World Health Organ 1991;69: $623-630$.

Manuscript received on 26 June 1997. Revised version accepted for publication on 10 April 1998.

RESUMEN En este estudio se evaluaron el rendimiento, la aceptabilidad y la conveniencia de la inmunización antitetánica extrainstitucional con un nuevo dispositivo hipodérmico de una sola dosis, el UniJect ${ }^{\circledR}$. De abril a junio de 1995, el dispositivo UniJect aprestado de

Vacunación antitetánica extrainstitucional por parteras empíricas bolivianas mediante dispositivos desechables de una sola dosis fábrica con una sola dosis de toxoide tetánico fue utilizado por 36 parteras tradicionales para vacunar a 2240 mujeres embarazadas durante las visitas domiciliarias prenatales de rutina en los distritos Norte, Ichilos y Warnes de Santa Cruz, Bolivia. Ya que el toxoide tetánico es termoestable, las parteras pudieron mantener los dispositivos UniJect sin refrigeración en sus hogares por un período de hasta un mes. Los UniJect se guardaron, transportaron y desecharon en portadores extrainstitucionales diseñados para reducir los riesgos de manipularlos y desecharlos de forma inadecuada.

Se recolectaron datos de las mujeres vacunadas, las parteras tradicionales y sus supervisores, mediante observación, cuestionarios y entrevistas realizadas después del estudio. Todos los grupos consideraron muy satisfactorios el rendimiento y la aceptabilidad del dispositivo UniJect. Las parteras tradicionales lo usaron de forma apropiada y cuidadosa; no hubo informes ni observaciones de mala utilización, reúso o puntazos accidentales. Se mencionaron, como ventajas del dispositivo, que no requiere montaje, asegura la esterilidad y reduce el desperdicio de vacuna que a veces ocurre con las ampollas de dosis múltiples. Además, todo el procedimiento se simplifica porque los dispositivos pueden almacenarse y transportarse sin necesidad de hielo. 